\title{
On Left Centralizers of Semiprime $\Gamma$-Rings
}

\author{
K. K. Dey ${ }^{*}$ and A. C. Paul \\ Department of Mathematics, Rajshahi University, Rajshahi-6205, Bangladesh
}

Received 5 October 2011, accepted in final revised form 23 January 2012

\begin{abstract}
Let $\mathrm{M}$ be a semiprime $\Gamma$-ring satisfying an assumption $x \alpha y \beta z=x \beta y \alpha z$ for all $x, y, z \in M, \alpha$, $\beta \in \Gamma$. In this paper, we prove that a mapping $T: M \rightarrow M$ is a centralizer if and only if it is a centralizing left centralizer. We also show that if $T$ and $S$ are left centralizers of $M$ such that $T(x) \alpha x+x \alpha S(x) \in Z(M)$ (the center of $M$ ) for all $x \in M, \alpha \in \Gamma$, then both $T$ and $S$ are centralizers.
\end{abstract}

Keywords: Semiprime $\Gamma$-ring; Left (right) centralizer; Centralizer; Commuting mapping; Centralizing mapping: Extended centroid.

(c) 2012 JSR Publications. ISSN: 2070-0237 (Print); 2070-0245 (Online). All rights reserved.

doi: http://dx.doi.org/10.3329/jsr.v4i2.8691 J. Sci. Res. 4 (2), 349-356 (2012)

\section{Introduction and Preliminaries}

Let $M$ and $\Gamma$ be additive abelian groups. $M$ is called a $\Gamma$-ring if for all $x, y, z \in M, \alpha, \beta \in \Gamma$ the following conditions are satisfied :

(i) $x \beta y \in M$,

(ii) $(x+y) \alpha z=x \alpha z+y \alpha z, x(\alpha+\beta) y=x \alpha y+x \beta y, x \alpha(y+z)=x \alpha y+x \alpha z$,

(iii) $(x \alpha y) \beta z=x \alpha(y \beta z)$.

Every ring is a $\Gamma$-ring and many notions on the ring theory are generalized to $\Gamma$-rings. Let $M$ be a $\Gamma$-ring. A subring $I$ of $M$ is an additive subgroup which is also a $\Gamma$-ring. A right ideal of $M$ is a subring $I$ such that $I \Gamma M \subset I$. Similarly a left ideal can be defined. If $I$ is both a right and a left ideal then we say that $I$ is an ideal.

Let $S$ be a subset of $M$. If $x \alpha y+y \alpha x \in S$, for all $x, y \in S, \alpha \in \Gamma$, then $S$ is called a Jordan subring of $M$.

The commutator $x \alpha y-y \alpha x$ will be denoted by $[x, y]_{\alpha}$. We know that $[x \beta y, z]_{\alpha}=$ $[x, z]_{\alpha} \beta y+x \beta[y, z]_{\alpha}+x[\beta, \alpha]_{z} y$ and $[x, y \beta z]_{\alpha}=y \beta[x, z]_{\alpha}+[x, y]_{\alpha} \beta z+y[\beta, \alpha]_{x} z$. We take an assumption $\left(^{*}\right) x \beta z \alpha y=x \alpha z \beta y$ for all $x, y, z \in M$ and $\alpha, \beta \in \Gamma$. Using the assumption the

\footnotetext{
* Corresponding author: kkdmath@yahoo.com
} 
basic commutator identities reduce to $[x \beta y, z]_{\alpha}=[x, z]_{\alpha} \beta y+x \beta[y, z]_{\alpha}$ and $[x, y \beta z]_{\alpha}=$ $y \beta[x, z]_{\alpha}+[x, y]_{\alpha} \beta z$.

Throughout, $M$ denotes a $\Gamma$-ring with center $Z(M)$. $M$ is said to be semiprime if $x \Gamma M \Gamma x=0$ implies $x=0$, it is prime if $x \Gamma M \Gamma y=0$ implies $x=0$ or $y=0$. An additive mapping $T: M \rightarrow M$ is called a left (right) centralizer if $T(x \alpha y)=T(x) \alpha y(T(x \alpha y)=x \alpha T(y)$ ) for all $x, y \in M, \alpha \in \Gamma$. If $a \in M$, then $L_{\mathrm{a}}(x)=a \alpha x$ and $R_{\mathrm{a}}(x)=x \alpha a,(x \in M, \alpha \in \Gamma)$ define a left centralizer and a right centralizer of $M$, respectively. An additive mapping $T: M \rightarrow M$ is called a centralizer if $T(x \alpha y)=T(x) \alpha y=x \alpha T(y)$ for all $x, y \in M, \alpha \in \Gamma$. A mapping $f: M$ $\rightarrow M$ is called centralizing (skew centralizing) if $[f(x), x]_{\alpha} \in Z(M)(f(x) \alpha x+x \alpha f(x) \in Z(M))$ for all $x \in M, \alpha \in \Gamma$, in particular, if $[f(x), x]_{\alpha}=0(f(x) \alpha x+x \alpha f(x)=0)$ for all $x \in M, \alpha \in \Gamma$, then it is called commuting (skew-commuting). Obviously every commuting (skewcommuting) mapping $f: M \rightarrow M$ is centralizing (skew centralizing). We recall if $f: M \rightarrow$ $M$ is commuting, then $[f(x), y]_{\alpha}=[x, f(y)]_{\alpha}$ for all $x, y \in M, \alpha \in \Gamma$. A mapping $f: M \rightarrow M$ is called central if $f(x) \in Z(M)$ for all $x \in M$.

The theory of centralizers in rings is well established. Many mathematicians worked on centralizers of rings and found out some remarkable results. The theories of Banach algebras and $C^{*}$-algebra with centralizers are established by many authors.

Bresar [1-3] studied centralizing mappings with derivation in prime rings. Mayne [4] worked on centralizing automorphisms of prime rings. Recently, Vukman [5-7] and Zalar [8] studied on centralizer of semiprime rings and 2-torsion free semiprime rings. Samman and Chaudhry [9] established the necessary and sufficient condition for a mapping to be a centralizer. If two left centralizers $T$ and $S$ of a semiprime ring $R$ satisfying $T(x) x+$ $x S(x) \in Z(R)$ for all $x \in R$, then they also prove that both $T$ and $S$ are centralizers. Haque and Paul [10] worked on Jordan centralizers on a $\Gamma$-ring with certain assumption. For the extended centroid we refer to $[11,12]$. They proved that every Jordan left centralizer on a 2-torsion free semiprime $\Gamma$-ring is a left centralizer. They also proved that every Jordan centralizer on a 2-torsion free semiprime $\Gamma$-ring satisfying a certain condition is a centralizer.

In this paper, we develop the results of [9] in Gamma rings. Our results are the generalizations of the results of Samman and Chaudhry [9]. The results in this paper for left centralizers are also true for right centralizers because of left-right symmetry.

\section{Left Centralizers on Semiprime $\Gamma$-rings}

In this section, we prove our main results.

Theorem 2.1 Let $S$ be a set and $M$ be a semiprime $\Gamma$-ring. If the functions $f$ and $g$ of $S$ into $M$ satisfy

$f(s) \alpha x \beta g(t)=g(s) \alpha x \beta f(t)$ for all $s, t \in S, x \in M, \alpha, \beta \in \Gamma$,

then there exist idempotent elements $e_{1}, e_{2}, e_{3} \in C$, the extended centroid on $\mathrm{M}$ and an invertible $k \in C$ such that $e_{i} \alpha e_{j}=0$ for $i \neq j, e_{1}+e_{2}+e_{3}=1$ and $e_{1} \alpha f(s)=k \beta e_{1} \alpha g(s)$, $e_{2} \alpha g(s)=0, e_{3} \alpha f(s)=0$ hold for all $s \in S, \alpha, \beta \in \Gamma$. 
Proof. Obviously, the identity holds in case $x$ is an element from $C(M)$, the central closure of $M$. Thus there is no loss of generality in assuming that $M$ is centrally closed. Let $A=$ $M \Gamma f(s) \Gamma M$ and $B=M \Gamma g(s) \Gamma M$. We have $A^{\perp}=p \Gamma M$ and $B^{\perp}=q \Gamma M$ for some idempotent elements $p, q \in C$. We set $e_{1}=(1-p) \alpha(1-q), e_{2}=(1-p) \alpha q$ and $e_{3}=p$. Clearly $e_{i}$ 's $(i=1$, 2,3 ) are mutually orthogonal idempotent elements with sum 1 . Since $q \alpha g(s) \in B^{\perp}, s \in S$, $\alpha \in \Gamma$, we have $q \alpha g(s) \beta x \delta q \alpha g(s)=0$, which implies $q \alpha g(s)=0$. Hence $e_{2} \alpha g(s)=0, s \in S$, $\alpha \in \Gamma$. Similarly we see that $e_{3} \alpha f(s)=0, s \in S, \alpha \in \Gamma$.

We note that $\left(e_{1} \alpha A\right)^{\perp}=\left(e_{1} \alpha B\right)^{\perp}=\left(1-e_{1}\right) \alpha M$, that is, $\left(e_{1} \alpha A\right)^{\perp}=\left(e_{1} \alpha B\right)^{\perp}=\left(1-e_{1}\right) \Gamma M$. Hence $E=e_{2} \Gamma A \oplus\left(1-e_{1}\right) \Gamma M$ is an essential ideal of $M$. Define $\phi: E \rightarrow M$ by $\phi\left(e_{1} \alpha\left(\sum_{i=1}^{3} x_{i} \beta f\left(s_{i}\right) \delta y_{i}\right)+\left(1-e_{1}\right) \lambda r\right)=e_{1} \alpha\left(\sum_{i=1}^{3} x_{i} \beta g\left(s_{i}\right) \delta y_{i}\right)+\left(1-e_{1}\right) \lambda r$.

In order to show that $\phi$ is well defined, we suppose that

$e_{1} \alpha\left(\sum_{i=1}^{3} x_{i} \beta f\left(s_{i}\right) \delta y_{i}\right)=0$. Consequently $e_{1} \alpha\left(\sum_{i=1}^{3} x_{i} \beta f\left(s_{i}\right) \delta y_{i}\right) \gamma z \lambda g(t)=0$ holds for all $z \in M, t \in S, \alpha, \beta, \delta, \gamma, \lambda \in \Gamma$.

Since by (1) we have $f\left(s_{i}\right) \delta y_{i} \gamma z \lambda g(t)=g\left(s_{i}\right) \delta y_{i} \gamma z \lambda f(t)$, it follows that $e_{1} \alpha\left(\sum_{i=1}^{3} x_{i} \beta g\left(s_{i}\right) \delta y_{i}\right) \gamma z \lambda f(t)=0$ for all $z \in M, t \in S, \alpha, \beta, \delta, \gamma, \lambda \in \Gamma$.

Thus the elements $e_{1} \alpha\left(\sum_{i=1}^{3} x_{i} \beta g\left(s_{i}\right) \delta y_{i}\right)$ lies in $A^{\perp}$. Since $A^{\perp}=p \Gamma M$ and $e_{1}=(1-p) \alpha(1-$ $q)$, it follows that $e_{1} \alpha\left(\sum_{i=1}^{3} x_{i} \beta g\left(s_{i}\right) \delta y_{i}\right)=0$. This proves that $\phi$ is well defined.

Clearly $\phi$ is an $M_{\Gamma}$-module homomorphism. Then there exist $k \in C$ such that $\phi(u)=k \beta u$ for every $u \in E, \beta \in \Gamma$. Hence $e_{1} \alpha f(s)=k \beta e_{1} \alpha g(s)$ for all $s \in S, \alpha, \beta \in \Gamma$. It remains to prove that $k$ is invertible. Note that $k \Gamma E=e_{1} \Gamma B \oplus\left(1-e_{1}\right) \Gamma M$. Since $e_{1} \Gamma B \oplus\left(1-e_{1}\right) \Gamma M$ is an essential ideal (namely $\left.\left(e_{1} \Gamma B\right)^{\perp}=\left(1-e_{1}\right) \Gamma M\right), k$ can not be a divisor of zero. Consequently, $C$ is the extended centroid of $M, k$ is invertible. The proof is complete.

Theorem 2.2. Let $M$ be a 2-torsion free semiprime $\Gamma$-ring satisfying the condition (*) and $U$ be a Jordan subring of $M$. If an additive mapping $F$ of $M$ into itself is centralizing on $U$, then $F$ is commuting on $U$.

Proof: A linearization of $[F(x), x]_{\alpha} \in Z$ gives $[F(x), y]_{\alpha}+[F(y), x]_{\alpha} \in Z$ for all $x, y \in U, \alpha \in \Gamma$.

Replacing $y$ by $x \beta x$,

$[F(x), x \beta x]_{\alpha}+[F(x \beta x), x]_{\alpha} \in Z$. Since $[F(x), x]_{\alpha} \in Z$, we have $[F(x), x \beta x]_{\alpha}$

$=x \beta[F(x), x]_{\alpha}+[F(x), x]_{\alpha} \beta x$

$=[F(x), x]_{\alpha} \beta x+[F(x), x]_{\alpha} \beta x=2[F(x), x]_{\alpha} \beta x$. Thus

$2[F(x), x]_{\alpha} \beta x+[F(x \beta x), x]_{\alpha} \in Z$ for all $x \in U, \alpha, \beta \in \Gamma$.

By assumption $[F(x \beta x), x \beta x]_{\alpha} \in Z$, for all $x \in U, \alpha, \beta \in \Gamma$. That is

$[F(x \beta x), x]_{\alpha} \beta x+x \beta[F(x \beta x), x]_{\alpha} \in Z$. 
Now fix $x \in U$ and let $z=[F(x), x]_{\alpha}, u=[F(x \beta x), x]_{\alpha}$. We must show that $z=0$. By (2) we have

$$
\begin{aligned}
& 0=[F(x), 2 z \beta x+u]_{\alpha} \\
& =2 z \beta[F(x), x]_{\alpha}+2[F(x), z]_{\alpha} \beta x+[F(x), u]_{\alpha}=2 z \beta z+[F(x), u]_{\alpha}
\end{aligned}
$$

Thus $[F(x), u]_{\alpha}=-2 z \beta z$

According to (3) we have $0=[F(x), u \beta x+x \beta u]_{\alpha}=[F(x), u]_{\alpha} \beta x+u \beta[F(x), x]_{\alpha}+[F(x)$, $x]_{\alpha} \beta u+x \beta[F(x), u]_{\alpha}$, applying (4) we then get $-4 z \beta z \beta x+2 z \beta u=0$.

Thus $z \beta u=2 z \beta z \beta x$. Multiplying (4) by $z \beta$ and using the last relation we obtain

$-2 z \beta z \beta z=z \beta[F(x), u]_{\alpha}=[F(x), z \beta u]_{\alpha}-[F(x), z]_{\alpha} \beta u=[F(x), z \beta u]_{\alpha}$

$=[F(x), 2 z \beta z \beta x]_{\alpha}=2 z \beta z \beta[F(x), x]_{\alpha}+[F(x), 2 z \beta z]_{\alpha} \beta x=2 z \beta z \beta z$. Hence $z \beta z \beta z=0$.

Since the center of a semiprime $\Gamma$-ring contains no nonzero nilpotent elements, we conclude that $z=0$. This proves the theorem.

Theorem 2.3 Let $T$ be a centralizing left centralizer of a semiprime $\Gamma$-ring $M$ satisfying the condition (*). Then $T$ is commuting.

Proof. If $M$ is 2-torsion free, then $T$ is commuting follows from Theorem 2.3 by taking $U=M$ in it. If $\mathrm{M}$ is not a 2-torsion free semiprime $\Gamma$-ring, then

$$
\begin{aligned}
& 2[T(x), x]_{\alpha}=0 \text { for all } x \in M, \alpha \in \Gamma(5) \text { and } \\
& 2\left([T(x), y]_{\alpha}+[T(y), x]_{\alpha}\right)=0 \text { for all } x, y \in M, \alpha \in \Gamma .
\end{aligned}
$$

By assumption $[T(x), x]_{\alpha} \in Z(M)$. Linearizing this, we get

$[T(x), y]_{\alpha}+[T(y), x]_{\alpha} \in Z(M)$ for all $x, y \in M, \alpha \in \Gamma$. easily

Using (5) - (7) and the hypothesis that $[T(x), x]_{\alpha} \in Z(M)$, the following identity follows

$$
[T(x), x \beta y+y \beta x]_{\alpha}+[T(y), x \beta x]_{\alpha}=0 \text { for all } x, y \in M, \alpha, \beta \in \Gamma .
$$

Replacing $y$ by $y \delta x$ in (8), we get $[T(x), x \beta y \delta x+y \delta x \beta x]_{\alpha}+[T(y \delta x), x \beta x]_{\alpha}=0$, which gives $(x \beta y+y \beta x) \delta[T(x), x]_{\alpha}+[T(x), x \beta y+y \beta x]_{\alpha} \delta x+T(y) \delta[x, x \beta x]_{\alpha}+[T(y), x \beta x]_{\alpha} \delta x=0$ for all $x, y \in M, \alpha, \beta, \delta \in \Gamma$. Combining this with (8), we get $(x \beta y+y \beta x) \delta[T(x), x]_{\alpha}=0$ for all $x, y \in M, \alpha, \beta, \delta \in \Gamma$, which gives $(x \beta y-y \beta x+2 y \beta x) \delta[T(x), x]_{\alpha}=0$, for all $x, y \in M, \alpha, \beta$, $\delta \in \Gamma$. Thus $(x \beta y-y \beta x) \delta[T(x), x]_{\alpha}=0$ for all $x, y \in M, \alpha, \beta, \delta \in \Gamma$. In particular, (replacing $\mathrm{y}$ by $T(x)$ and $\delta$ by $\alpha)(x \beta T(x)-T(x) \beta x) \delta[T(x), x]_{\alpha}=-[T(x), x]_{\alpha} \beta[T(x), x]_{\alpha}=0$ for all $x \in M$, $\alpha, \beta \in \Gamma$. Since a semiprime $\Gamma$-ring has no nontrivial central nilpotents, therefore $[T(x), x]_{\alpha}$ $=0$ for all $x \in M, \alpha \in \Gamma$.

Theorem 2.4 Let $T$ be a centralizing left centralizer of a semiprime $\Gamma$-ring $M$ satisfying the condition (*), then $T$ is a centralizer of $M$. 
Proof. We have $T(x \alpha y)=T(x) \alpha y$ for all $x, y \in M$ and $\alpha \in \Gamma$. We now show that $T(x \alpha y)=$ $x \alpha T(y)$ for all $x, y \in M, \alpha \in \Gamma$. Since $T$ is a centralizing left centralizer of $M$, therefore by Theorem 2.3, it is commuting. Thus $[T(x), x]_{\alpha}=0$ for all $x \in M, \alpha \in \Gamma$. That is, $T(x) \alpha x-$ $x \alpha T(x)=0$ for all $x \in M, \alpha \in \Gamma$. Linearizing this, we get $T(x) \alpha y+T(y) \alpha x-y \alpha T(x)-x \alpha T(y)$ $=0$ for all $x, y \in M, \alpha \in \Gamma$.

Replacing $y$ by $x \beta y$ in the last identity, we get

$0=T(x) \alpha x \beta y+T(x \beta y) \alpha x-x \beta y \alpha T(x)-x \alpha T(x \beta y)=T(x) \alpha x \beta y+T(x) \beta y \alpha x-x \beta y \alpha T(x)$

$-x \alpha T(x) \beta y=(T(x) \alpha x-x \alpha T(x)) \beta y+T(x) \beta y \alpha x-x \alpha y \beta T(x)=T(x) \beta y \alpha x-x \alpha y \beta T(x)$.

That is,

$T(x) \beta y \alpha x-x \beta y \alpha T(x)=0$ for all $x, y \in M, \alpha, \beta \in \Gamma$.

Taking $S=M, s=t=x, f(x)=T(x)$ and $g(x)=x$ in (9) and applying Theorem 2.1 to (9) we conclude that there exist idempotent elements $e_{1}, e_{2}, e_{3} \in C$ and an invertible $p \in C$ such that $e_{i} \alpha e_{j}=0$ for $i \neq j, e_{1}+e_{2}+e_{3}=1$ and $e_{1} \alpha T(x)=p \beta e_{1} \alpha x, e_{2} \alpha x=0$ and $e_{3} \alpha T(x)=0$ for all $x \in M, \alpha, \beta \in \Gamma$. Now $e_{2} \alpha x=0$ implies $x \alpha e_{2}=0$. Thus $T\left(x \alpha e_{2}\right)=T(0)$, which gives $T(x) \alpha e_{2}=T(0) \alpha 0=0$. That is, $T(x) \alpha e_{2}=0$ or $e_{2} \alpha T(x)=0$. Thus

$$
\left.T(x)=e_{1}+e_{2}+e_{3}\right) \alpha T(x)=e_{1} \alpha T(x)=p \beta e_{1} \alpha x .
$$

That is, $T(x)=p \beta e_{1} \alpha x$ for all $x \in M, \alpha \in \Gamma$. Thus $T(x) \alpha y-x \alpha T(y)=p \beta e_{1} x \delta y-x \alpha p \beta e_{1} \delta y$ $=p \beta e_{1} \alpha x \delta y-p \beta e_{1} \alpha x \delta y=0$. That is,

$$
T(x) \alpha y=x \alpha T(y) \text { for all } x, y \in M, \alpha \in \Gamma \text {. }
$$

$$
(T(x \alpha y)-T(x) \alpha y) \beta z \gamma(T(x \alpha y)-T(x) \alpha y)=0 .
$$

By the semiprimeness of $M$, we have, $T(x \alpha y)-T(x) \alpha y=0$. This implies $T(x \alpha y)=$ $T(x) \alpha y$. Thus $T(x \alpha y)=T(x) \alpha y=x \alpha T(y)$. This shows that $T$ is a centralizer.

Remark 2.5 Obviously every centralizer is commuting because $T(x \alpha x)=T(x) \alpha x=x \alpha T(x)$ for all $x \in M, \alpha \in \Gamma$, and hence is a centralizing left centralizer. Thus we have the following corollary.

Corollary 2.6 A mapping $T$ of a semiprime $\Gamma$-ring $M$ satisfying the condition (*) is a centralizer if and only if it is a centralizing left centralizer. Let $T$ be a commuting left centralizer of a semiprime $\Gamma$-ring, then $T(x) \beta[x, y]_{\alpha}=x \beta[T(x), y]_{\alpha}$ holds for all $x, y \in M, \alpha$, $\beta \in \Gamma$.

Proof. Since $T$ is commuting, therefore $[T(x), x]_{\alpha}=0$ for all $x \in M, \alpha \in \Gamma$.

Linearizing (11), we get

$$
[T(x), y]_{\alpha}+[T(y), x]_{\alpha}=0 \text { for all } x, y \in M, \alpha \in \Gamma \text {. }
$$

Replacing $y$ by $x \beta y$ in (12) and using (12), we get $0=[T(x), x \beta y]_{\alpha}+[T(x \beta y), x]_{\alpha}=$ $[T(x), x \beta y]_{\alpha}+[T(x) \beta y, x]_{\alpha}=[T(x), x]_{\alpha} \beta y+x \beta[T(x), y]_{\alpha}+[T(x), x]_{\alpha} \beta y+T(x) \beta[y, x]_{\alpha}=$ $x \beta[T(x), y]_{\alpha}-T(x) \beta[x, y]_{\alpha}$.

That is, $x \beta[T(x), y]_{\alpha}-T(x) \beta[x, y]_{\alpha}=0$ for all $x, y \in M, \alpha, \beta \in \Gamma$. 
Thus $\quad T(x) \beta[x, y]_{\alpha}=x \beta[T(x), y]_{\alpha}$ for all $x, y \in M, \alpha, \beta \in \Gamma$.

Remark 2.7 If $T$ is a central left centralizer of a prime $\Gamma$-ring $M$, then either $T=0$ or $M$ is commutative. This is because $T(x) \beta[x, y]_{\alpha}=x \beta[T(x), y]_{\alpha}$ gives $T(x) \beta[x, y]_{\alpha}=0$. Replacing $y$ by $y \delta z$ in the last identity and using it, we get $T(x) \beta y \delta[x, z]_{\alpha}=0$ for all $x, y, z \in M$. Since $M$ is prime, therefore $T(x)=0$ or $[x, z]_{\alpha}=0$ for all $x, z \in M, \alpha \in \Gamma$. That is, $T=0$ or $M$ is commutative.

Theorem 2.8. Let $M$ be a semiprime $\Gamma$-ring satisfying the condition (*) and $T$ and $S$ be left centralizers of $M$ such that

$T(x) \alpha x+x \alpha S(x) \in Z(M)$ for all $x \in M, \alpha \in \Gamma$.

Then $T$ and $S$ are both centralizers.

Proof. Linearizing (13), we get

$T(x) \alpha y+T(y) \alpha x+x \alpha S(y)+y \alpha S(x) \in Z(M)$ for all $x, y \in M, \alpha \in \Gamma$.

Thus $[T(x) \alpha y+T(y) \alpha x+x \alpha S(y)+y \alpha S(x), x]_{\beta}=0$, which gives

$[T(x) \alpha y+T(y) \alpha x+x \alpha S(y), x]_{\beta}=-[y \alpha S(x), x]_{\beta}$ for all $x, y \in M, \alpha, \beta \in \Gamma$.

Replacing $y$ by $y \beta x$ in (14), we get $T(x) \alpha y \beta x+T(y \beta x) \alpha x+x \alpha S(y \beta x)+y \beta x \alpha S(x)=$ $T(x) \alpha y \beta x+T(y) \beta x \alpha x+x \alpha S(y) \beta x+y \beta x \alpha S(x)=(T(x) \alpha y+T(y) \alpha x+x \alpha S(y)) \beta x+$ $y \alpha x \beta S(x) \in Z(M)$.

Thus $[(T(x) \alpha y+T(y) \alpha x+x \alpha S(y)) \beta x+y \alpha x \beta S(x), x]_{\beta}=0$ for all $x \in M, \alpha, \beta \in \Gamma$. This implies that

$[T(x) \alpha y+T(y) \alpha x+x \alpha S(y), x]_{\beta} \beta x+[y \beta x \alpha S(x), x]_{\beta}=0$ for all $x, y \in M, \alpha, \beta \in \Gamma$.

Using (15), from (16) we get

$-[y \alpha S(x), x]_{\beta} \beta x+[y \alpha x \beta S(x), x]_{\beta}=0$ for all $x, y \in M, \alpha, \beta \in \Gamma$.

Since $[y \alpha S(x) \beta x, x]_{\beta}=[y \alpha S(x), x]_{\beta} \beta x$, therefore (17) gives $0=-[y \alpha S(x) \beta x, x]_{\beta}+$ $[y \alpha x \beta S(x), x]_{\beta}=[y \alpha(x \beta S(x)-S(x) \beta x), x]_{\beta}=\left[y \alpha[x, S(x)]_{\beta}, x\right]_{\beta}=y \alpha\left[[x, S(x)]_{\beta}, x\right]_{\beta}+[y$, $x]_{\beta} \alpha[x, S(x)]_{\beta}$.

Thus

$y \alpha\left[[x, S(x)]_{\beta}, x\right]_{\beta}+[y, x]_{\beta} \alpha[x, S(x)]_{\beta}=0$ for all $x, y \in M, \alpha, \beta \in \Gamma$.

Replacing $y$ by $z \lambda y$ in (18) and using (18), we get $0=z \lambda y \alpha\left[[x, S(x)]_{\beta}, x\right]_{\beta}+[z \lambda y$, $x]_{\beta} \alpha[x, S(x)]_{\beta}=z \lambda y \alpha\left[[x, S(x)]_{\beta}, x\right]_{\beta}+z \lambda[y, x]_{\beta} \alpha[x, S(x)]_{\beta}+[z, x]_{\beta} \lambda y \alpha[x, S(x)]_{\beta}=$ $[z, x]_{\beta} \lambda y \alpha[x, S(x)]_{\beta}$.

That is, $\quad[z, x]_{\beta} \lambda y \alpha[x, S(x)]_{\beta}=0$ for all $x, y, z \in M, \alpha, \beta, \lambda \in \Gamma$.

In particular, $[S(x), x]_{\beta} \lambda y \alpha[x, S(x)]_{\beta}=0$ which, by semiprimeness of $M$, implies $[S(x), x]_{\beta}=0$. Thus $S$ is a commuting left centralizer and, by Theorem 2.2, is a centralizer.

We now show that $T$ is commuting. By hypothesis and by the assumption, we have

$0=[T(x) \alpha x+x \alpha S(x), x]_{\beta}=[T(x), x]_{\beta} \alpha x+x \alpha[S(x), x]_{\beta}=[T(x), x]_{\beta} \alpha x$. That is, 
$[T(x), x]_{\beta} \alpha x=0$ for all $x, y \in M, \alpha, \beta \in \Gamma$.

From (15), we get $[T(x) \alpha y+T(y) \alpha x, x]_{\beta}=[-x \alpha S(y)-y \alpha S(x), x]_{\beta}$. Thus $T(x) \alpha[y, x]_{\beta}$

$+[T(x), x]_{\beta} \alpha y+[T(y), x]_{\beta} \alpha x=-x \alpha[S(y), x]_{\beta}-y \alpha[S(x), x]_{\beta}-[y, x]_{\beta} \alpha S(x)=$

$-x \alpha[y, S(x)]_{\beta}-[y, x]_{\beta} \alpha S(x)=x \alpha[S(x), y]_{\beta}+[x, y]_{\beta} \alpha S(x)$.

That is, for all $x, y \in M, \alpha, \beta \in \Gamma$

$T(x) \alpha[y, x]_{\beta}+[T(x), x]_{\beta} \alpha y+[T(y), x]_{\beta} \alpha x=x \alpha[S(x), y]_{\beta}+[x, y]_{\beta} \alpha S(x)$.

Again by hypothesis, we get

$0=[T(x) \alpha x+x \alpha S(x), y]_{\beta}=T(x) \alpha[x, y]_{\beta}+[T(x), y]_{\beta} \alpha x+[x, y]_{\beta} \alpha S(x)+x \alpha[S(x), y]_{\beta}$.

That is, for all $x, y \in M, \alpha, \beta \in \Gamma$

$-T(x) \alpha[y, x]_{\beta}+[T(x), y]_{\beta} \alpha x=-[x, y]_{\beta} \alpha S(x)-x \alpha[S(x), y]_{\beta}$.

Adding (21) and (22), we get

$[T(x), x]_{\beta} \alpha y+[T(y), x]_{\beta} \alpha x+[T(x), y]_{\beta} \alpha x=0$ for all $x, y \in M, \alpha, \beta \in \Gamma$.

Replacing $y$ by $y \beta T(x)$ in (23) and using (20), we get

$$
\begin{aligned}
0 & =[T(x), x]_{\beta} \alpha y \beta T(x)+[T(y \beta T(x)), x]_{\beta} \alpha x+[T(x), y \beta T(x)]_{\beta} \alpha x \\
& =[T(x), x]_{\beta} \alpha y \beta T(x)+[T(y) \beta T(x), x]_{\beta} \alpha x+[T(x), y \beta T(x)]_{\beta} \alpha x \\
& =[T(x), x]_{\beta} \alpha y \beta T(x)+T(y) \beta[T(x), x]_{\beta} \alpha x+[T(y), x]_{\beta} \beta T(x) \alpha x+[T(x), y]_{\beta} \beta T(x) \alpha x \\
& =-[T(y), x]_{\beta} \alpha x \beta T(x)-[T(x), y]_{\beta} \alpha x \beta T(x)+[T(y), x]_{\beta} \beta T(x) \alpha x+[T(x), y]_{\beta} \beta T(x) \alpha x \\
& =[T(y), x]_{\beta} \alpha[T(x), x]_{\beta}+[T(x), y]_{\beta} \alpha[T(x), x]_{\beta} .
\end{aligned}
$$

That is

$[T(y), x]_{\beta} \alpha[T(x), x]_{\beta}+[T(x), y]_{\beta} \alpha[T(x), x]_{\beta}=0$ for all $x, y \in M, \alpha, \beta \in \Gamma$.

Replacing $y$ by $y \lambda x$ in (24) and using (23), we get

$$
\begin{aligned}
0 & =[T(y \lambda x), x]_{\beta} \alpha[T(x), x]_{\beta}+[T(x), y \lambda x]_{\beta} \alpha[T(x), x]_{\beta} \\
& =[T(y) \lambda x, x]_{\beta} \alpha[T(x), x]_{\beta}+[T(x), y \lambda x]_{\beta} \alpha[T(x), x]_{\beta} \\
& =[T(y), x]_{\beta} \lambda x \alpha[T(x), x]_{\beta}+y \lambda[T(x), x]_{\beta} \alpha[T(x), x]_{\beta}+[T(x), y]_{\beta} \lambda x \alpha[T(x), x]_{\beta} \\
& =\left([T(y), x]_{\beta} \lambda x+[T(x), y]_{\beta} \lambda x\right) \alpha[T(x), x]_{\beta}+y \lambda[T(x), x]_{\beta} \alpha[T(x), x]_{\beta} \\
& =-[T(x), x]_{\beta} \lambda y \alpha[T(x), x]_{\beta}+y \lambda[T(x), x]_{\beta} \alpha[T(x), x]_{\beta} .
\end{aligned}
$$

Thus

$[T(x), x]_{\beta} \lambda y \alpha[T(x), x]_{\beta}=y \lambda[T(x), x]_{\beta} \alpha[T(x), x]_{\beta}$ for all $x, y \in M, \alpha, \beta, \lambda \in \Gamma$.

Replacing $y$ by $x \alpha y$ in (25) and using (20), we get $x \alpha y \lambda[T(x), x]_{\beta} \alpha[T(x), x]_{\beta}=[T(x)$, $x]_{\beta} \alpha x \alpha y \lambda[T(x), x]_{\beta}=0$.

That is, 
$x \alpha y \lambda[T(x), x]_{\beta} \alpha[T(x), x]_{\beta}=0$ for all $x, y \in M, \alpha, \beta, \lambda \in \Gamma$,

which gives $T(x) \beta x \alpha y \lambda[T(x), x]_{\beta} \alpha[T(x), x]_{\beta}=0$.

Further, replacing $y$ by $T(x) \beta y$ in (26), we get $x \alpha T(x) \beta y \lambda[T(x), x]_{\beta} \alpha[T(x), x]_{\beta}=0$. Combining the last two identities, we get $(T(x) \beta x-x \beta T(x)) \alpha y \lambda[T(x), x]_{\beta} \alpha[T(x), x]_{\beta}=0$. That is, $[T(x), x]_{\beta} \gamma y \lambda[T(x), x]_{\beta} \alpha[T(x), x]_{\beta}=0$, which gives $[T(x), x]_{\beta} \alpha[T(x), x]_{\beta} \alpha y \lambda[T(x)$, $x]_{\beta} \alpha[T(x), x]_{\beta}=0$. Since $M$ is semiprime, therefore,

$[T(x), x]_{\beta} \alpha[T(x), x]_{\beta}=0$ for all $x \in M, \alpha, \beta \in \Gamma$.

Using (23), from (21) we get $[T(x), x]_{\beta} \alpha y \lambda[T(x), x]_{\beta}=0$, which by semiprimeness of $M$ implies $[T(x), x]_{\beta}=0$. Thus $T$ is a commuting left centralizer and hence by Theorem 2.2, $T$ is a centralizer.

Taking $S=T$ in Theorem 2.7, we get the following corollary.

Corollary 2.9. Let $T$ be a skew centralizing left centralizer of a semiprime $\Gamma$-ring $M$ satisfying the condition (*). Then $T$ is a centralizer.

The following corollary is also obvious.

Corollary 2.10. Let $T$ and $S$ be left centralizers of a semiprime $\Gamma$-ring $M$ satisfying the condition $\left(^{*}\right)$ such that $T(x) \alpha x+x \alpha S(x)=0$ for all $x \in M, \alpha \in \Gamma$. Then both $T$ and $S$ are centralizers.

\section{References}

1. M. Brešar, Proc. Amer. Math. Soc. 114, 641 (1992).

2. M. Brešsar, J. Algebra 156, 385 (1993). http://dx.doi.org/10.1006/jabr.1993.1080

3. M. Brešsar, Proc. Amer. Math. Soc. 120, 709 (1994).

4. J.H. Mayne, Canad. Math. Bull. 19, 113 (1976). http://dx.doi.org/10.4153/CMB-1976-017-1

5. J. Vukman, Comment. Math. Univ. Carolinae 38, 231 (1997).

6. J. Vukman, Comment. Math. Univ. Carolinae 40, 447 (1999).

7. J. Vukman, Comment. Math. Univ. Carolinae 42, 237 (2001).

8. B. Zalar, Comment. Math. Univ. Carolinae 34, 609 (1991).

9. M. S. Samman, M. A. Chaudhry, Int. J. Pure Appl. Math. 20 (4) 487 (2005).

10. M. F. Hoque and A. C. Paul, Int. Math. Forum 6 (13), 627 (2011).

11. M. A. Ozturk and Y. B. Jun, Commun. Korean Math. Soc. 15 (3), 469 (2000).

12. M. A. Ozturk and Y. B. Jun, Turk. J. Math. 25, 367 (2001).

13. K. K. Dey and A. C. Paul, J. Sci. Res. 3 (2), 331 (2011). http://dx.doi.org/10.3329/jsr.v3i2.7278

14. K. K. Dey and A. C. Paul, J. Sci. Res. 4 (1), 33 (2012). http://dx.doi.org/10.3329/jsr.v4i1.7911 